\title{
'Eye of the Beholder': Psychiatric Medical Reasoning, Narrative Humility, and Graphic Medicine
}

\author{
Sathyaraj Venkatesan ${ }^{1}$ and Arya Suresh ${ }^{2}$ \\ ${ }^{1}$ Associate Professor, Department of Humanities and Social Sciences, NIT Trichy, India \\ Email: sathyaiitk@gmail.com ORCID ID: oooo-0oo3-2138-1263 \\ ${ }^{2} \mathrm{PhD}$ Scholar, Department of Humanities and Social Sciences, NIT Trichy, India \\ Email: livearyalive@gmail.com ORCID ID: 0000-0002-3411-0094
}

\begin{abstract}
Within health humanities, graphic medicine narrates individual stories of patient experience in its interaction with the system of healthcare and its professionals. These autopathographies give a new perspective to the medicalized accounts of diseases and assign subjectivity to the voice which narrates its sufferings. From a medical perspective, clinical reasoning is an important step in the treatment of any disease and a procedure that determines the course of the upcoming treatment. However, in psychiatry, clinical reasoning is a problematic terrain with its lack of external validating criteria and increased reliance on non-somatic symptoms of the disease. In many instances, the authority of biomedical knowledge takes over clinical reasoning and completely denies the individuality of a mental patient and his or her story. This research article attempts to investigate how individual stories and experiences are undermined in psychiatric clinical reasoning discourses and recognizes the importance of empathy and compassion in medical listening through a close reading of select graphic memoirs on bipolar disorder. Citing certain panels from Rachel Lindsay's $R x$ (2018) and Ellen Forney's Marbles: Mania, Depression, Michelangelo and Me (2012), this study analyses the pitfalls of clinical reasoning in psychiatry and the widening gap of doctor-patient communication in such facilities. Interweaving the theory of Sayantani Das Gupta's Narrative humility with instances taken from the above mentioned texts this article discusses the imperative need to restore empathy in medical listening.
\end{abstract}

Keywords: Graphic medicine, psychiatry, medical listening, clinical reasoning, psychiatric gaze, narrative humility, empathy

\section{Introduction}

In the movie Patch Adams (1998), the American biopic made on the life of Hunter Doherty "Patch" Adams, the protagonist effusively states the reason why he wants to become a doctor. "I wanna listen. I wanna really listen to people" (Shadyac, 1998, oo:16:02). The film is a display of the ingrained, long-standing lack of empathy and compassion among doctors to patients and Patch's response to this myopic institutional legacy of medical education. Needless to say, the stereotypical image of a doctor as a white-coated human, rigid in his mannerisms, with penurious listening skills and sparse and urgent communication is what most of the patients encounter in a healthcare setting. Apart from all the external impediments resulting from lack of infrastructure in a medical facility, cultural barriers between doctor and patient or shortage of staff in the hospital, the unspoken dogma laid out in the profession regarding the dynamics of communication between a doctor and a patient plays a significant part in the power structure within the exchanges. Not only free interaction with the patients make the diagnosis and treatment uncomplicated for the doctor

(C) AesthetixMS 2021. This Open Access article is published under a Creative Commons Attribution Non-Commercial 4.0 International License (http://creativecommons.org/licenses/by-nc/4.o/), which permits non-commercial re-use, distribution, and reproduction in any medium, provided the original work is properly cited. For citation use the DOI. For commercial re-use, please contact editor@rupkatha.com. 
but "patients who understand their doctors are more likely to acknowledge health problems, understand their treatment options, modify their behaviour accordingly, and follow their medication schedules" (Travaline et al., 2005, p.13).

In any medical encounter, diagnosis is one of the most important steps, ever so in the era of the pandemic. It is established through a series of interviews with the patient, discussing the history of illnesses, examining the patient physically, results of diagnostic imaging and finally connecting the dots using the available data. Further, clinical reasoning of the medical practitioner is a skill applied in diagnosis which is the "ability to integrate and apply different types of knowledge, to weigh evidence, critically think about arguments and to reflect upon the process used to arrive at a diagnosis" (Andersen, 2006, p.1-4). With its inordinate stress on diagnostic accuracy based on these objective criteria the person in which the disease is diagnosed is often overlooked. Particularly, when the process of diagnosis in psychiatry is considered, a challenge is that it "cannot be confirmed as correct in the same way as in other areas of medicine because there are no physical examination signs or test results that indicate the pathology of most mental illnesses" (Gupta et al, 2019, p.50). Psychiatrists rely on DSM (Diagnostic and Statistical Manual of Mental Disorders) and patient's history to arrive at a conclusion. Even then, the epistemic considerations of psychiatry prioritize "a roadmap that will enable clinicians to understand their patients' problems” (Lemoine, 2012) over a straight-to-cure action plan of treatment. Such roadmaps will have to overcome the inclination towards objective data. Moving away from the empirical paradigms of diagnosis and treatment the psychiatrist should take an effort to decipher the "intersubjectivity", what J. Benjamin describes as "the field of intersection between two subjectivities, the interplay between two different subjective worlds"(1990, p. 34). Building upon the concept of intersubjectivity, Gupta et al. define "intersubjective knowing" as an "interpersonal learning that occurs between people who are relating to each other" a concept that is "dynamic and reciprocal, meaning that the actions, speech, interpretations, and history of each actor influences those of the others and evolves throughout the interaction" (2019, p.50). Needless to say, intersubjective knowing demands a detailed understanding between the psychiatrist and the patient and empathy for the patient's existence.

\section{Situating empathy in patient care and response to illness memoirs}

One of the prominent suggestions of the 'People-Centred Health Care: A policy framework' released by WHO in 2007 was 'improving health care quality and safety and enhancing the people's experience of care requires attention not only to health system design but also to the focus and process of patient care' (p. 3). As reiterated by WHO in this policy document, the human interactions within the healthcare system are as important as the science, technology, knowledge and pedagogy in medicine. After all, the paramount objective of any doctor-patient communication is to improve patient's health and to optimize the healthcare setting. Research conducted by Kim, Kaplowitz and Johnstone reveals that patient satisfaction and treatment compliance were directly related to a physician's empathic behaviour. Also, physician's "affective empathy" and "sense of partnership" towards the patient elicit satisfaction and compliance in the latter (2004, p.238). In the peculiar case of psychiatric treatment, the tenets of doctor-patient interactions are prominent than any other speciality of medicine because it presents with specific aspects and unique challenges. One such challenge of mental health disciplines, when compared to other disciplines in medicine, is that "the care is delivered to patients who have varying degrees of awareness, beliefs, and understanding about their illness. The illness itself can affect their ability to trust, understand, or make judgments to arrive at a decision" (Harbishettar et al., 2019, p.776). However, Priebe et al. 
(2011) formulated five guiding principles for effective communication in psychiatric clinical practice: (1) a focus on the patient's concerns, in the sense that patient's complaints and wishes have to guide the consultation; (2) positive regard and personal respect towards a patient who is valued as a person, regardless of the observed behaviour; (3) appropriate involvement of patients in decision making, respecting the level of involvement preferred by the patients; (4) genuineness with a personal touch, knowing how to be in the relationship, not only what and how to do; and (5) the use of a psychological treatment model to induce therapeutic change. These guidelines thrust on the autonomy of the patient as an individual and to invest some faith in their subjectivity.

Similar to the medical dilemma regarding the varying degree of reliability on a mental patient's account of his or her illness, the process of autobiographical retelling of one's psy-journey is looked upon with equal suspicion in many cases. There are reservations even when it comes to theorizations in autobiographical writing of people with affective disorders. As Sidonie Smith and Julia Watson doubt the authority of such a narrator thus: "the life narrator describing a breakdown from an asserted position of recovery is always suspect" (p.145). Conversely, Jeffrey Berman in his Mad Muse disagrees with Smith and Watson on their contention that "those who suffer from mood and thought disorders lack the authority, permission, or strength to write about their lives" (p. 5). Assertive of his position, he points out the example of Kay Redfield Jamison and others who suggested that "people who suffer from mood disorders are not depressed or manic all the time: there are periods of lucidity that make writing possible" (p.5). Subsequently, in his response to the need for empathy towards such memoirs Berman quotes himself from Narcissism and the Novel (1990) thus: "although empathy has often been dismissed as a touchy-feely concept that is antithetical to rigorous critical thinking, empathy allows us to begin to understand the other, particularly, the suffering other" (p.5). Thus, the feeling of empathy bridges the emotional gap between the narrator and the reader and brings the reader closer to the experience.

\section{Graphic Medicine: Dismantling the rigid structures through comics}

Ian Williams, one of the pioneers of the Graphic medicine movement, defines the scope of the field thus: "graphic medicine combines the principles of narrative medicine with an exploration of the visual systems of comic art, interrogating the representation of physical and emotional signs and symptoms within the medium" (Czerwiec et al. 2015, p.1). Graphic memoirs as a whole "open up new and troubled spaces" (Whitlock, 2006, p.976) and when this canvas thematically employ illness, medicine and body on its narrative ground have "emerged to fill a niche for patients and doctors" (Green \& Myers, p.574). Simply put, graphic medicine narratives make use of the medium of comics to narrate the deep subjective experience as a patient, caregiver or doctor about a wide range of issues related to illness, disability, body and healthcare. It is, as Susan Squier defines in Graphic Medicine Manifesto(2015), "a movement for change that challenges the dominant methods of scholarship in healthcare, offering a more inclusive perspective of medicine, illness, disability, caregiving and being cared for" (Czerweic et. Al, p.2). Medicine has been historically subordinating the voice of the suffering patient with its innate empiricism, hegemonic prescriptivism and decorum of its practising class. Graphic medicine movement, the prominent figures of which include doctors, nurses, professors, scholars, librarians and many more, engages in innovative ways in the curriculum of medical education in several universities (e.g. Penn State University, USA) to introduce comics and "solve ethical dilemmas...caused by poor communication" and to initiate a culture of conversation on "how doctors and patients tell stories to one another and what they hear" (Czerweic et al., 2015, p.9). It also encourages the medical students to be better listeners to the patients and appreciate the latter's essential dispositions. These pathographies present before 
doctors a person as a patient in its entirety. Graphic medicine helps "to avoid the trope of God complex in doctors" (Blenk, 2019, 06:50) and "to connect with the idea that their patients really are humans" (07:17).

The array of critically acclaimed graphic pathographies include Harvey Pekar and Joyce Brabner's Our Cancer Year (1994), Brian Fies' Mom's Cancer (2006), Frederik Peeters' Blue Pills (2008), Sarah Leavitt's Tangles (2010), Ken Dahl's Monsters (2009), David Small's Stiches (2010), Ian Williams' The Bad Doctor (2014), M. K. Czerwiec's Taking Turns: Stories from HIV/AIDS Care Unit 371 (2017), and Georgia Webber's Dumb: Living without a Voice (2018) many of which are critical of the healthcare system. A glance into the oeuvre of graphic medicine proves the variety of its narrative voices, perspectives and the strength of perspectival volte-face in medical narratives. Notably, Ian Williams' The Bad Doctor (2014) and The Lady Doctor (2019) are two works written from the perspective of a medical practitioner, sketching out the challenges and dilemmas within the profession. Dr Iwan James, the autobiographical persona of Ian Williams, in the Bad Doctor is burdened by his professional responsibilities to the point where it becomes an enormous task for him to make decisions about others' lives. At the same time, his vulnerable side as an OCD patient, the concept of the wounded healer is revealed through the narration. Subsequently, its sequel Lady Doctor explores a variety of issues in healthcare such as the politics of rural practice, the frustrations of a doctor dealing with adamant patients, the problems of gender in medical practice and many other issues in the healthcare scenario. These graphic memoirs accommodate the vulnerable voices within the system of medicine irrespective of their subjective identities as a patient, a doctor, a nurse or a caregiver.

The striking similarities of Rachel Lindsay's $R x(2018)$ and Ellen Forney's Marbles: Mania, Depression, Michelangelo $\mathcal{E} M e$ (2012), the two graphic medicine texts selected for our study are that both of them deal with the growing pains of the artist's journey through bipolar disorder and their common concern about how the medication would affect their life as an artist. On the other hand, starkly different are the approaches of their psychiatrists' towards their respective patients. While Lindsay following a mental breakdown is admitted to a psychiatric facility, Forney navigates through thirteen years of ups and downs of bipolar, maintaining the status of an outpatient. Lindsay's tryst with the system of psychiatry, her expectations of the human relations in the system and Forney's repetitive but supportive consultations with her psychiatrist Karen represent two different approaches towards patient care and clinical reasoning. Representative of two distinct approaches to patients, these graphic enunciations are testimonies to what is and what is not expected of psychiatrists in the patient-doctor interface.

\section{Narrative humility towards patient testimonials}

"Does some kind of al/chemical transformation need to occur before the mentally ill can be heard? And in whom does it need to take place?" asked Catherine Prendergast (Price, 2011, p.8). Indeed, Rachel Lindsay repeats this question through $R x(2018)$, a critically acclaimed memoir in the genre of graphic medicine, on a short period of Lindsay's bipolar life and admission into a psychiatric facility. Her stay in the hospital brought her tete-a-tete with the realities of a mental patient/bipolar identity and its diminutive validation from the perspective of the mental healthcare system.

In a striking set of panels against prominent jet black gutters (n.p.), Lindsay emotionally recounts her expectations of an empathic psychiatrist. She wants one person of authority to validate her experience of illness, a panel where the empathetic doctor tosses away the clipboard of medical notes in front of a tearful Lindsay. Considering the notable use of the clipboard as a metaphor of 
clinical reasoning in the text, the tossing of the clipboard can be interpreted as an act of turning away from a critical medical gaze. Illustrative of this instance, Sayantani DasGupta's concept that clinicians should witness the stories of suffering persons with narrative humility is about admitting the fact that "the patient's story, at least initially, belongs entirely to him" (2008, p.980). Narrative humility, as Das Gupta puts it, acknowledges "that our patients' stories are not objects that we can comprehend or master, but rather dynamic entities that we can approach and engage with, while simultaneously remaining open to their ambiguity and contradiction, and engaging in constant self-evaluation and self-critique about issues such as our role in the story, our expectations of the story, our responsibilities to the story, and our identifications with the story- how the story attracts or repels us because it reminds us of any number of personal stories" (2008, p.981). DasGupta quotes medical educator and philosopher Craig Irvine to strengthen her take on narrative humility by asking the health practitioners to be open with "the sense of humility toward that which we do not know-the face of the Other, the face we cannot know but to which we are responsible" (2008, p.980). Lindsay longed for a doctor to listen to her with empathy and narrative humility, with intersubjectivity, so that she can reinvent her identity, agency and locate her personhood within the institution.

Also, Lindsay seeks her lost agency in the second panel where she is stripped off of her selfhood as an independent adult in the psychiatric facility. The decision of her conscious self to quit the job she disliked was considered as an erratic decision from mood episode by her family and the establishment. In addition to all these feelings, she kept a strong disgust towards doctors who did not save her from her doom, howsoever she tried to evade hospitalization through proper medication and consultation. In general, these panels indicate the presence of stifled voices in this episteme, the voice of the sufferer, which is to be considered as an important factor in medical reasoning. As Jose Medina states, “The willingness to put one's cognitive perspective [and one's affective self] in relation to that of others-calibrating the different cognitive forces, impulses, and compulsions one is exposed to-is the path to the epistemic virtues" (Medina, 2013, p. 51). To reach the best accessible approximation of the mental health situation of the patient, the psychiatrist has to be ready for empathetic listening and should actively participate in intersubjective knowing. Lindsay wishes her doctor to identify her peculiar condition and its context through intersubjective knowing and cultivate narrative humility.

\section{Escaping the clipboard}

Rachel Lindsay portrays her travails to negotiate different realities the medical narratives imposed upon her in the psychiatric ward in the chapter ' $11 N$ ' of $R x$. . The narrative voice of the doctor goes like this through continuing panels on the clipboard in one of these pages (Lindsay, 2018, n.p.): 'Patient describes hospitalization as a "mind-blowing act of betrayal", 'she does not believe she is manic...but rather being punished by her parents for making a brave, independent decision without consulting them'. Even when Lindsay acknowledges the existence of her mental illness and been compliantly taking pills, she questions the complete denial of agency of a mental patient while she or he undergoes the process of medical reasoning. She succinctly portrays her attempt to escape the clipboard of medical records by making dynamic use of the conventions of comics. In ' $11 \mathrm{~N}$ ', a series of splash page panels modelled in the form of a doctor's clipboard elucidates the doctor's clinical perspective on Lindsay's life in the psychiatric ward. In one of those brilliant clipboard splash pages, in the last of the nine square panels describing her oscillating moods in a manic episode, Lindsay tries to escape the clipboard. With an efficacious use of the verbo-visual affordances of comics, she strides her leg across the rim of the panel by looking around whether 
she will be caught or not. Lindsay lands upon another white page of free space only to be caught by an enormous hand and put back onto the clipboard again (2018, n.p.).

On examining the larger picture, the lack of agency and being identified as an unreliable narrator by the system of medicine is prevalent in the treatment of affective disorders. As a testimony to this, Lindsay, in one of her presentations delivered at Harvard Country Library, divulges the comments written on the medical notes she acquired from her psychiatric facility. She smiles at how her aspiration to show her cartoon portfolio to The New Yorker has been described as "delusional" in the medical notes made during her treatment period (MK Czerweic, 2019). It is the blatant ignorance of the patient's identity, interests and life that prompts the medical practitioners to be dismissive of his or her aspirations. The general neglect and sometimes apathy towards narratives of mental patients is epistemic violence. Thus Braslow, Brekke \& Levenson (2020) critique the existing paradigms of the psychiatric treatment system and the need for a holistic approach inclusive of the social body of the patient thus:

"despite compelling arguments in medicine and psychiatry against clinical reductionism, we parse patients' diseases into signs and symptoms that yield to pharmacological and/or time-limited psychosocial treatments that address parts of psychiatric disease while failing to address the most human and tragic ravages of SMI (Serious Mental Illness). By mistaking the parts for the whole, clinical psychiatry has aided and abetted the social alienation; social, medical, and psychiatric abandonment; and neglect inflicted on those with SMI over the last half-century" (E1)

Further, Rachel Lindsay comments on sanity as a "performance" in a psychiatric institution (2018, n.p.). The patient has to comply with a set of rules and fall in line with the decorum of the institution in a ritualistic manner even though these rules might negate the very sense of self of the patient. She regards sanity as a performance in this context where she performs on a stage to the music, tapping her feet and moving her body in front of a dark silhouette of the audience. The audience, in the next panel, is revealed as doctors wearing glasses, with a serious look on their face scribbling on their clipboards. Consequently, Sanity is being judged on the basics of the theatrics here, reducing the individual order of the human mind to general notions of insanity. The medical reasoning in psychiatry is also influenced by the ableist notions of society, one rather called sanism. Sanism as defined by AMA Journal of Ethics is "an irrational prejudice against people with mental illness, is of the same quality and character as other irrational prejudices such as racism, sexism, homophobia, and ethnic bigotry that cause (and are reflected in) prevailing social attitudes"(AMA, 2013). This prejudice mediated by the formal knowledge of medicine coupled with the preconceived stereotypes about the mentally ill and scepticism towards personal narratives on mental illness lead towards bracketing of illness experiences.

\section{"I am your doctor, not your parent": Empathetic listening in Marbles}

In Ellen Forney's Marbles, one witness yet another face of the empathetic doctor-patient relationship, the reason why Forney dedicates her book partly to her psychiatrist. Forney's consultation segments with her psychiatrist Karen interspersed with episodes of manic highs and depressive lows constitute the narrative. Throughout the narrative trajectory of the work, there occur tremendous changes in their interaction, the trust Forney has in Karen strengthening with each consultation. Karen is an iconic empath as a psychiatrist and listens to her patient with utmost patience, a relationship that runs for around thirteen years. 
In one of those striking panels where Forney reveals her secretive consumption of pot (which she kept hidden from Karen) Karen retorts thus: "I am your doctor, not your parent" (2012, p.155). Essentially, Karen refrains from negotiating on Forney's moral agency as an individual even though the concept of the moral agency of a mentally ill person is problematic to consider. Emphasizing the unique therapeutic ways of psychiatry concerning treatment outcome, "reforming the patient's whole self or character..." as Radden (2002) remarks are "...akin to the responsibilities of raising children”. However, after allowing her perplexed mood to pass, Karen indicates the dangers of using Ecstasy drug to Forney not with a magisterial air of paternalism attributed to an authoritative figure but in a body language comparable to that of a confidante. Karen reaffirms the need for honesty in doctor-patient interactions for the good of the patient but here it is Forney who chooses to be defiant of the clinical stipulations by proclaiming to herself "This is mine to deal with. I don't have to talk about everything with Karen" (p.156). Overall, Karen's approach elucidates the need to confide in with the doctor and to set aside the metrics of morality in medical talks and clinical reasoning scenarios.

Consultation scenes in Marbles are shown mostly from medium long-shot, rendering a rather objective quality to this first-person graphic memoir. It establishes the monotony of these consultations but simultaneously chronicles the evolution of a certain warmth in the patientpsychiatrist relationship over years. The consultation room is set subverting the hierarchies of power in doctor-patient interactions because "the interplay of power relations in the therapeutic office is also significant" (Liddicoat, 2019, para.10). Interestingly, to facilitate an environment conducive to effective engagement with the patient, it is apposite to pay attention to the consultation space and its alignment. "Chairs should be evenly spaced and face the centre of the room or each other to mitigate power imbalances, hierarchies, or "otherness." There should also be no intervening desk between psychiatrist and patient" (Liddicoat, 2019, para.10) suggests design research on psychiatric consultation rooms to promote "greater sense of equality" and also reinforces "promote a higher therapeutic engagement by the patient and a better patientpsychiatrist alliance” (Liddicoat, 2019, para.10).

\section{Coda: Empathy and narrative humility in psychiatric clinical reasoning}

Graphic medicine is a genre that employs visual storytelling for narrating stories of healthcare. These stories critique or negotiate the socially accepted terrains of medical culture based on the first-hand experience of patients, healthcare professionals or caregivers. Clinical reasoning in psychiatry is different from the other specialities in medicine because of its lack of objective data based on which diagnostic assumptions are made often. On the other hand, this unique quality of psychiatric episteme demands a different set of skills from its practitioners in their interaction with patients. Doctor-patient interactions based on empathy towards the patient and knowledge of an intersection of subjectivities, otherwise called intersubjectivity knowing, will benefit the patient. By breaking the hegemonic power within these interactions, the communication between the patient and the psychiatrist can be made better, which can further result in the improvement of the patient's disease condition.

Rachel Lindsay's Rx and Ellen Forney's Marbles: Mania, Depression, Michelangelo and Me, apart from its thematic similarities, occupy two extremes of a spectrum when examined from the clinical reasoning point of view and in its representation of the psychiatrist-patient interface. Lindsay questions the episteme of psychiatry in its subjugation of her selfhood and identity through its practices. Several panels in $R x$ point out the patient's stifled voice in clinical reasoning, the power 
medical discourse exerts over patient's stories and how even their lucid moments are discarded for lack of empathy in the practitioners. Sayantani DasGupta's theory of narrative humility propounds the ability to listen to patient narratives keenly and accepting the importance of this story to value the patient's identity and sense of self. Lindsay's narration in its complaining tone, questions the lack of narrative humility among psychiatrists and their denial of her agency. Marbles, on the other hand, sets a good example of empathetic listening in psychiatry. This graphic memoir by Ellen Forney portrays how her decade long relationship with her psychiatrist helped her wade through the troubled waters of bipolar. The open conversations Karen, her psychiatrist, initiated about the medication, its effects on her body, her identity and her career as an artist reinstated her trust in treatment. These two narratives show how the approach of the psychiatrist can alter patient's perception of the episteme and thereby elicit better compliance from them. Thus, empathy and humane communications skills are paramount in psychiatric clinical reasoning and the doctorpatient relationship. By interweaving intersubjective knowing and narrative humility into the paradigm of psychiatry's discourse, a better clinical reasoning environment and communication culture can be nurtured among doctors.

\section{References}

Anderson KJ. (2006) Factors affecting the development of undergraduate medical students' clinical reasoning ability. $\mathrm{PhD}$ Thesis. The University of Adelaide;1-4.

Berman, J. (2019). Mad Muse: The Mental Illness Memoir in a Writer's Life and Work. Emerald Publishing Limited

Blenk, J. (Host). (2019, March 25). Issue \#9: Graphic Medicine and Disability in Comics (No.9) [Audio podcast episode]. In Comics Book Buds. https://open.spotify.com/episode/oJc82YgKJL2ssAZVY3n?si=OxtZKZI2QvesnTic-aFFyQ\&utm_source

Braslow JT, Brekke JS, Levenson J. (2021). Psychiatry's Myopia-Reclaiming the Social, Cultural, and Psychological in the Psychiatric Gaze. JAMA Psychiatry. 2021;78(4):349-350. doi:10.1001/jamapsychiatry.2020.2722

Czerwiec, M.K, et al. (2015). Graphic Medicine Manifesto. Pennsylvania: Pennsylvania State University Press.

DasGupta, S., (2008). Narrative Humility. The Lancet. Vol 371 March 22. Pp. 980-81

Forney, E. (2012). Marbles: Mania, Depression, Michelangelo \& Me. Avery

Green, M. J., and Myers, K.R. (2010) Graphic Medicine: Use of Comics in Medical Education and Patient Care. Bmj, vol. 340, no. maro3 2, pp. c863-c863., doi:10.1136/bmj.c863.

Harbishettar, V., Krishna, K.R., Srinivasa, P., Gowda, M. (2019). The enigma of doctor-patient relationship. Indian Journal of Psychiatry. 61(10): 776-781

Kim SS, Kaplowitz S, Johnston MV. (2004) The effects of physician empathy on patient satisfaction and compliance. Eval Health Prof;27:237-51.

Lemoine, M. (2012). How does a psychiatrist infer from an observed condition to a case of mental disorder?. Journal of Evaluation in Clinical Practice, 18, 5, 979-983.

Liddicoat, S. (2019). Strategies to facilitate a positive clinical encounter. Psychiatric Times 36(6). https://www.psychiatrictimes.com/view/strategies-facilitate-positive-clinical-encounter.

Lindsay, R. (2018). Rx. Grand Central Publishing 
Medina, J. (2013). Epistemologies of Resistance: Gender and Racial Oppression, Epistemic Injustice, and Resistant Imaginations. Oxford: Oxford University Press.

MK Czerweic. (2019, April 16). Rachel Lindsay Reclaiming the Patient Narrative in Graphic Medicine [Video]. YouTube. https://youtu.be/VK_BJwCQFUE

Perlin, M. L. (2013, October). Sanism and the Law. AMA Journal of Ethics. https://journalofethics.amaassn.org/article/sanism-and-law/2013-10

Price, M. (2011). Mad at School: Rhetorics of Mental Disability and Academic Life. University of Michigan Press

Priebe, S, Dimic, S, Wildgrube, C, Jankovic, J, Cushing, A, McCabe, R (2011). Good communication in psychiatry - a conceptual review. European Psychiatry 26, 403-407.

Radden, J. (2002). Psychiatric Ethics. Bioethics Sep; 16(5):397-411. doi: 10.1111/1467-8519.00298

Shadyac, T. (Director). (1998). Patch Adams [Film]. Universal Pictures

Whitlock, G. (2006). Autographics: The Seeing "I" of the Comics. MFS Modern Fiction Studies 52(4), 965979. doi:10.1353/mfs.2007.0013.

World Health Organization (2007). People-Centred Health Care: A policy framework. WHO Press: Geneva.

Sathyaraj Venkatesan is an Associate Professor of English in the Department of Humanities and Social Sciences at National Institute of Technology, Trichy, India. He is the author of six books and over ninety research publications that span African American literature, health humanities, graphic medicine, film studies, and other literary and culture studies disciplines. He is most recently coauthor of India Retold (New York: Bloomsbury, 2021).

Arya Suresh is a Ph.D. graduate student in the Department of Humanities and Social Sciences at National Institute of Technology, Trichy, India. Her research concentrates on graphic medicine, mad studies, and health humanities. 